\title{
Promoter expression landscape in skeletal muscle in hindlimb suspension and recovery model in rat
}

\author{
Guzel Gazizova \\ Kazan Federal University, Kazan, \\ Russia \\ grgazizova@gmail.com
}

Islam Nigmetzyanov

KFU, Kazan, Russia

islamka32@gmail.com

Ilia Akberdin

BIOSOFT.RU, LLC; Novosibirsk State

University, Novosibirsk, Russia

akberdinir@biosoft.ru

\author{
Sergei Pintus \\ BIOSOFT.RU, LLC; Institute of \\ Computational Technologies, \\ Novosibirsk, Russia \\ sspintus@biosoft.ru \\ Oksana Tyapkina \\ FRC KSC RAS, Kazan, Russia \\ antitoxin@icloud.com \\ Fedor Kolpakov \\ Institute of Computational \\ Technologies, Novosibirsk, Russia \\ fkolpakov@gmail.com
}

\author{
Ruslan Deviatiiarov \\ KFU, Kazan, Russia \\ ruselusalbus@gmail.com \\ Leniz Nurullin \\ FRC KSC RAS, Kazan, Russia \\ leniz2009@gmail.com \\ Oleg Gusev \\ KFU, Kazan, Russia \\ RIKEN, Yokohama, Japan \\ oleg.gusev@riken.jp
}

\begin{abstract}
Loss of muscle mass and function during long period of physical inactivity still remains a clinical problem for humanity because the significant reducing of life quality and increasing mortality. In recent studies global gene expression in atrophied and recovered skeletal muscles on different animal models was analyzed. However, previously whole-genome regulation of atrophy and following recovery at promoter and enhancer level has not been studied. To identify transcription initiation sites (TSS), and evaluate full-genome RNA expression at the level of individual promoters and enhancers during unloading and subsequent recovery in rats two types of muscles, "slow" (m. Soleus) and "fast" (m. EDL), were examined in rats in normal conditions, after 1,3 and 7 days of hindlimb suspension and following 1,3 and 7 days of recovery using CAGE (Cap Analysis of Gene Expression) method followed by Illumina HiSeq 2500 sequencing. We obtained 9971 unique CAGE clusters, among which 6377 were promoters associated with genes. Differential expression of genes and their promoter activity were strongly varied in $\mathrm{m}$. EDL and $\mathrm{m}$. soleus within suspension-recovery time course. This study provides the first systematic annotation of promoters landscape and genes activated in "fast" and "slow" muscle types under induced atrophy and following recovery in rats.
\end{abstract}

Keywords - muscle atrophy, muscle recovery, gene expression, promoter, $\mathrm{CAGE}$

\section{Motivation and aim}

Skeletal muscle represents adaptive system characterizing by plasticity. It is capable to remodeling in response to different external and internal stimuli. Mechanical unloading causes muscle atrophy but at the same time physical activity induces hypertrophy. Loss of muscle mass and function during long period of physical inactivity still remains a clinical problem for humanity because the significant reducing of life quality and increasing mortality. For resolving this task understanding molecular regulatory mechanisms of disuse muscle atrophy and recovery are required. In recent studies global gene expression in atrophied and recovered skeletal muscles on different animal models was analyzed. However, previously whole-genome regulation of atrophy and following recovery at promoter and enhancer level has not been studied. Here, for the first time we perform analysis of regulatory genome elements in skeletal muscles in hindlimb suspensionrecovery rat model. One of the most sensitive approaches for such analysis is Cap Analysis of Gene Expression method (CAGE), which allows determine transcription initiation sites (TSS) with up to one nucleotide precise, thus, evaluate the transcriptional activity of genes at promoter and enhancer level. In current study we aimed to identify transcription initiation sites (TSS), and evaluate full-genome RNA expression at the level of individual promoters and enhancers during unloading and subsequent recovery in rats.

\section{Methods}

Two types of muscles, "slow" (m. Soleus) and "fast" (m. EDL), were examined in rats in normal conditions, after 1, 3 and 7 days of hindlimb suspension and following 1, 3 and 7 days of recovery using CAGE (Cap Analysis of Gene Expression) method followed by Illumina HiSeq 2500 sequencing. After quality check and filtration, CAGE reads were mapped to the current rat genome assembly rn6 (2014) by using bwa and then clustered by python script. Further annotation of CAGE peaks, analysis of differential expression, and functional terms enrichment were proceeded through $\mathrm{R}$ environment.

\section{Results}

We obtained 9971 unique CAGE clusters, among which 6377 were promoters associated with genes. The remaining promoters were suggested to be unannotated transcripts, remotely located promoters of known genes and non-coding RNAs.

Differential expression of genes and their promoter activity were strongly varied in $\mathrm{m}$. EDL and $\mathrm{m}$. soleus within suspension-recovery time course: "slow" m. soleus has no significant changes of transcriptional activity up to 7 days of suspension, but drastically shifted during recovery, while "fast" m. EDL shows quick and stable response to the stress and fast recovery after placing in normal conditions. First atrophic changes were marked after 3 days of hindlimb suspension in soleus muscle and associated with upregulation of proteasome and peptidase complex genes. After the first day of recovery genes involved to transition between fast and slow fibers were activated in m. EDL. In addition, key transcription factors regulating the activity of specific muscle promoters were identified. Also enrichment of signaling pathways by differentially expressed genes was conducted.

This study provides the first systematic annotation of promoters landscape and genes activated in "fast" and "slow" muscle types under induced atrophy and following recovery in rats.

\section{ACKNOWLEDGMENT}

The reported study was funded by RFBR according to the research project No. 17-00-00243. 